\title{
Use of uterine fundal pressure maneuver at vaginal delivery and risk of severe perineal laceration
}

\author{
Philippus H. J. Hoogsteder • \\ Johanna M. A. Pijnenborg
}

Received: 28 July 2009/Accepted: 11 September 2009/Published online: 23 September 2009

(C) The Author(s) 2009. This article is published with open access at Springerlink.com

Matsuo et al. [1] published a very interesting, retrospective cohort study about the use of uterine fundal pressure maneuver at vaginal delivery and the risk of severe perineal laceration. A total of 661 vaginal deliveries were evaluated, in $39(5.9 \%)$ of these cases fundal pressure maneuver was applied.

The authors concluded the use of uterine fundal pressure during the second stage of labor increased the risk of severe perineal laceration, defined as a third- or fourth degree perineal tear. The risk of severe perineal laceration was $28.1 \%$ in the group that received fundal pressure versus $3.7 \%$ in the non-fundal pressure group, odds ratio 7.81 (95\% CI 3.33-18.3) and $P<0.001$. Even adjusted for previously described confounders like, primiparity, length of labor, episiotomy and vacuum extraction there was a significant higher rate of severe laceration in the fundal pressure group $[1,2]$. We compliment the authors for publishing these interesting data, since fundal pressure is frequently used during second stage as reported by Kline-Kaye et al. [3] but unfortunately seems to be underreported in medical literature.

Merhi and Awonuga [4] concluded in their sceptical reappraisal that the role of fundal pressure is understudied and remains controversial in the management of the second stage of labor, and caution should be exercised using this maneuver until it's proven to be safe and effective.

P. H. J. Hoogsteder $(\bowtie)$

Department of Obstetrics and Gynaecology,

Maastricht University Medical Centre, P.O. Box 5800,

6202 AZ Maastricht, The Netherlands

e-mail: p.hoogsteder@og.unimaas.nl

J. M. A. Pijnenborg

Department of Obstetrics and Gynaecology,

TweeSteden Hospital, Tilburg, The Netherlands
An interesting review published by Berghella et al. recommends against routinely providing fundal pressure in the termination of the second stage of labor. They conclude there are no significant better outcomes studied in women who received fundal pressure, while these women were less satisfied with the second stage of labor. The authors state the use of obstetric interventions should be studied and associated with the highest risk of safety and effectiveness, with avoidance of less safe and less effective interventions $[5,6]$.

Although the study by Matsuo et al. is the first to determine the risk for severe perineal laceration associated with the use of fundal pressure, we feel there are some concerns about the interpretation of these data.

As already stated by the authors, the study is limited to the lack of information about the indication of fundal pressure, the number and the duration of the maneuver, and the position of the fetal presenting part. In our opinion the indication of fundal pressure is important for the interpretation of the data. When fundal pressure is performed due to prolonged second stage fetal presentation plays an important role in the risk of severe perineal laceration.

As described by Pearl et al. [7], deliveries with the fetal occiput in posterior position had a significant higher incidence of severe perineal laceration than presentation with the occiput in anterior position. Unfortunately the study by Matsuo et al. was unable to obtain information about the presentation. On the contrary, when fundal pressure is used to accelerate second stage due to fetal distress with a normal fetal presentation, the risk of severe perineal laceration would probably be less when compared to abnormal presentation and prolonged second stage.

A total of nine severe perineal lacerations in the fundal pressure group of 39 women seems to be a rate of severe 
perineal laceration of $23.1 \%$ in contrary to the $28.1 \%$ as described by the authors. Although the authors clearly demonstrate that in the synergistic effects of episiotomy, vacuum extraction and fundal pressure, fundal pressure indeed seems to increase the risk of severe perineal laceration, we should know why these deliveries were instrumental deliveries to fully understand these results.

Api et al. [8] recently published a randomized controlled trial about the role of fundal pressure on the duration of the second stage of labor. This study randomized a total of 197 women between fundal pressure concomitant with each uterine contraction and normal pushing during second stage, without a medical indication. The authors concluded that fundal pressure was ineffective in shortening the second stage of labor, with no adverse outcomes of mother or child. There was no significant difference in severe perineal laceration between both groups. However, there seemed to be a trend towards a shorter second stage in a subgroup of nulliparous women. It would be interesting to compare the outcome of the study of Matsuo et al. comparing nulliparous women only.

The association described between a larger maternal body weight gain during pregnancy and the use fundal pressure maneuver of $11.16 \pm 0.4$ versus $10.05 \pm 0.16 \mathrm{~kg}$ in the non-fundal pressure group seems to be a statistically significance without clinical relevance [1].

The contribution of the trial by Matsuo et al. is important for further studies regarding fundal pressure related to the rate of adverse events. Documentation of fundal pressure when applied with clear indication seems to be the first step towards insight in the obstetrical practice of fundal pressure.
Conflict of interest statement We declare that we have no conflict of interest.

Open Access This article is distributed under the terms of the Creative Commons Attribution Noncommercial License which permits any noncommercial use, distribution, and reproduction in any medium, provided the original author(s) and source are credited.

\section{References}

1. Matsuo K, Shiki Y, Yamasaki M, Shimoya K (2009) Use of uterine fundal pressure maneuver at vaginal delivery and risk of severe perineal laceration. Arch Gynecol Obstet (Epub ahead of print)

2. Lowder JL, Burrows LJ, Krohn MA, Weber AM (2007) Risk factors for primary and subsequent anal sphincter lacerations: a comparison of cohorts by parity and prior mode of delivery. Am J Obstet Gynecol 196(4):344

3. Kline-Kaye V, Miller-Slade D (1990) The use of fundal pressure during the second stage of labor. J Obstet Gynecol Neonatal Nurs 19(6):511-517

4. Merhi ZO, Awonuga AO (2005) The role of uterine fundal pressure in the management of the second stage of labor: a reappraisal. Obstet Gynecol Surv 60:599-603

5. Berghella V, Baxter JK, Chauhan SP (2008) Evidence-based labor and delivery management. Am J Obstet Gynecol 199(5):445-454

6. Cox J, Cotzias CS, Siakpere O, Osuagwu FI, Holmes EP, PatersonBrown S (1999) Does an inflatable obstetric belt facilitate spontaneous vaginal delivery in nulliparae with epidural analgesia. Br J Obstet Gynaecol 106:1280-1286

7. Pearl ML, Roberts JM, Laros RK, Hurd WW (1993) Vaginal delivery from the persistent occiput posterior position. Influence on maternal and neonatal morbidity. J Reprod Med 38(12):955961

8. Api O, Balcin ME, Ugurel V, Api M, Turan C, Unal O (2009) The effect of uterine fundal pressure on the duration of the second stage of labor: a randomized controlled trial. Acta Obstet Gynecol Scand 88(3):320-324 